\title{
ORIGINAL
}

\section{EFECTO PROTECTOR DEL APOYO SOCIAL EN LA MORTALIDAD EN POBLACIÓN ANCIANA: UN ESTUDIO LONGITUDINAL}

Manel Nebot (1), Jose Manuel Lafuente (2), Zoa Tomás (1), Carme Borrell (1) y Josep Ferrando (1)

(1) Institut Municipal de Salut Pública de Barcelona

(2) Escola Universitaria de Infermería de Sant Joan de Deu. Universitat de Barcelona

\section{RESUMEN}

Fundamentos: El objetivo principal del estudio es analizar la relación entre el apoyo social y la mortalidad en una cohorte de población de 60 años o más, residente en Barcelona y no institucionalizada, observada durante el período de seguimiento de 1996 a 1999, con el objetivo de analizar la relación entre e apoyo social y la mortalidad en las personas que constituyen la cohorte.

Métodos: En 1996 se realizó una entrevista telefónica a 755 personas (316 hombres y 439 mujeres) que habían sido entrevistadas en la encuesta de salud de Barcelona de 1992, y que en ese año tenían 60 años o más. En el cuestionario se recogían variables socio-demográficas, de morbilidad y estado de salud, de apoyo social y de red social. Se han registrado los fallecimientos ocurridos entre 1996 y 1999. Se ha utilizado la regresión logística para analizar la asociación del apoyo social con la mortalidad.

Resultados: El número de defunciones durante este período fue de $55(5,9 \%$ de las mujeres y $9,2 \%$ de los hombres). Entre las variables que reflejan el apoyo social para los hombres, únicamente la situación de convivencia mostró en el análisis bivariado una asociación significativa con la mortalidad, que fue superior para los hombres que vivían con otros familiares pero no si vivían con su esposa o compañera $(\mathrm{OR}=3,7$; $\mathrm{IC}$ $95 \%$ 1,4-9,6). Entre las mujeres, la existencia de apoyo vecinal, el tamaño de la red familiar, el número de contactos con la red comunitaria y la situación de convivencia se asociaron a la mortalidad en el análisis bivariado, aunque en la regresión logística multivariada, únicamente el apoyo vecinal $(\mathrm{OR}=3,6$; IC:1,1-11,1) mantuvo una asociación significativa.

Conclusiones: Los resultados muestran una asociación de las variables de apoyo social con la mortalidad. La relación entre las diversas variables de apoyo social, y los posibles mecanismos de prevención deberían ser abordados en futuros estudios.

Palabras clave: Apoyo social. Estudios longitudinales. Mortalidad. Ancianos.

Correspondencia:

Manel Nebot

Servicio de Promoción de la Salud

Instituto Municipal de Salud Pública

Pl. Lesseps, 1

08023 Barcelona

Correo electrónico: mnebot@imsb.bcn.es
ABSTRACT

\section{Preventive Effect of Social Support on mortality Among Elderly Population: A Longitudinal Study}

Background: The main objective of this study is to analyze the relationship between social support and the mortality among a non-institutionalized population cohort age 60 or above residing in Barcelona studied throughout the 1996-1999 monitoring period for the purpose of analyzing the relationship between the social support and the mortality of this cohort.

Methods: In 1996, a telephone survey was conducted of 755 individuals (316 males and 439 females) who had been surveyed in the 1992 Barcelona health survey and who, were age 60 or above that year. The questionnaire included socio-demographic, morbility and health condition, social support and social network-related variables. The deaths during the 1996-1999 period were recorded. Logic regression was used for analyzing the social support- mortality relationship.

Results: The deaths for the period in question totaled 55 individuals $(5.9 \%$ of the females and $9.2 \%$ of the males). Among the variables reflecting social support, for the males solely the situation of living with someone was found, in the bivariate analysis, to have a significant relationship to the mortality, which was higher for those males who lived with other family members, but not with their wives or female partner $(\mathrm{OR}=3.7$; IC $95 \%$ 1.4-9.6). Among the females, the existence of support from neighbors, the size of the family network, the number of contacts with the community network and the situation of living with someone else were related to the mortality in the bivariate analysis, although in the multivariate logic regression, solely the support of neighbors (OR=3.6; IC:1.1-11.1) was found to have a significant relationship.

Conclusions: The results are consistent with prior studies revealing a relationship between the social support variables and the mortality. The relationship between the different social support variables and the possible prevention mechanisms should be taken up in future studies.

Keywords: Social support. Social network. Non-institutionalized elderly population. Mortality 


\section{INTRODUCCIÓN}

En las últimas décadas, diversos estudios epidemiológicos han puesto de manifiesto la asociación entre la falta de apoyo social y la mortalidad, en estudios de seguimiento en la población general ${ }^{1-5} \mathrm{y}$ en investigaciones centradas en la población anciana ${ }^{6-17}$. Sin embargo, la medida e incluso la definición del apoyo social ha sido abordada de formas muy distintas. En términos generales, el apoyo social se podría definir como el apoyo recibido o interacción entre las personas (familiares, amigos, vecinos y miembros de organizaciones sociales) con la finalidad de dar y recibir ayuda de diversa índole, básicamente apoyo espiritual, emocional, instrumental e informativo ${ }^{6-7 ; 18-19}$. Por otro lado, el término red social englobaría las características de las personas o grupos de los que se recibe este apoyo, ya sean las características estructurales (número de vínculos en la red, tamaño de la red), cantidad y calidad de la interacción (frecuencia e intensidad de las interacciones entre los miembros de la red) y características de la clase de servicios que proporciona la red (apoyo informativo, afectivo y tangible $)^{1 ; 6 ; 18}$. Entre los mecanismos que explicarian la relación del apoyo social sobre la salud se han propuesto las influencias sobre los comportamientos que tienen un impacto sobre la misma, mecanismos de tipo psicológico, como mejora de la autoestima, autoeficacia o la capacidad de afrontar las situaciones problemáticas, y los mecanismos fisiológicos, estudiados fundamentalmente en animales, y que podrían incluir cambios en el sistema inmunitario o en la reactividad cardiovascular, entre otros ${ }^{20}$.

Los escasos estudios realizados en nuestro país, entre los que destacan los de los municipios de Leganés $^{21}$ y de Córdoba ${ }^{22}$, han abordado básicamente la descripción de la red social y de la ayuda recibida por las personas mayores como una aproximación al estudio de las necesidades sociosanitarias, mientras que en el anterior estudio realizado en la muestra de la Encuesta de Salud de Barcelona de 1992 (ESB92) se observó una correlación entre la falta de apoyo emocional y la peor salud percibida entre los hombres ${ }^{23}$. Para estudiar el efecto del apoyo social en la salud entre las personas ancianas se diseñó el presente estudio, cuyo objetivo es analizar la asociación entre las variables de apoyo social y las características de la red social con la mortalidad general tras un seguimiento de cinco años.

\section{SUJETOS Y MÉTODOS}

Se trata de un estudio longitudinal en el que se ha estudiado la mortalidad sucedida entre 1996 y 1999 en una muestra de la población general entrevistada con un cuestionario telefónico en 1996. La población elegible en 1996 la constituían las personas que habían sido entrevistadas en su domicilio en 1992 como parte de la ESB92 y que tenían en el momento de la encuesta 60 o más años y no estaban institucionalizados. De los 1.170 sujetos entrevistados en 1992, en 1996 se pudo obtener respuesta válida a un cuestionario telefónico en 755 individuos, que representan un $64,5 \%$ del total. El resto corresponde a 244 personas perdidas $(20,8 \%)$ y 171 fallecidas en el intervalo $(14,7 \%)$. El cuestionario telefónico se diseñó a partir de la ESB92, incluyendo variables sociodemográficas, estado de salud percibido y variables de apoyo social y de red social.

El apoyo social se recogió con cuatro grupos de variables: a) las variables apoyo vecinal y apoyo emocional, procedentes de la ESB92 ${ }^{24}$ que recogían la existencia de personas en el vecindario con las que poder contar para pequeños favores, $y$ de personas de las que pudieran obtener apoyo emocional, respectivamente; b) preguntas relativas a la red comunitaria, incorporadas en un estudio de seguimiento realizado en $1994^{25}$, que recogían la asistencia regular a la iglesia, al mercado o a algún lugar céntrico del barrio; c) preguntas relativas al número de familiares y amigos disponibles (red fami- 
liar), procedentes del estudio «Envejecer en Leganés» ${ }^{26} ;$ y d) el estado civil y la situación de convivencia. Debido a la elevada colinealidad de estas dos variables, se combinaron en una única variable (situación de convivencia) con tres categorías: las personas casadas o conviviendo en pareja (en pareja), las personas solteras, viudas, separadas o divorciadas que vivían solas (solos autónomos), y las de similar estado civil conviviendo con otros familiares (solos dependientes).

La información relativa a la mortalidad se obtuvo enlazando nuestra cohorte con el registro de mortalidad del Instituto Municipal de Salud Pública de Barcelona. Se realizó un análisis bivariado para identificar las variables asociadas con la mortalidad, y un análisis multivariado mediante regresión logística para poder determinar la influencia independiente de estas variables. Este análisis se realizó mediante la construcción de diferentes modelos en los que se incluyeron en primer lugar todas las variables independientes, las cuales fueron posteriormente retiradas o mantenidas en dichos modelos en función de su relevancia conceptual, así como de la colinealidad que presentaban entre ellas, la significación de los coeficientes y el cambio significativo de la deviance ${ }^{27}$. Se realizaron modelos de regresión logística para hombres y mujeres por separado, dada la interacción existente entre el sexo y las variables de apoyo social. Excepto la edad, que se trató como una variable continua, el resto de las variables explicativas fueron tratadas de forma categórica. Con el fin de ajustar por el estado de salud se analizó la influencia del estado de salud percibido y de las variables de necesidad de ayuda en el cuidado personal y en las actividades de la vida diaria. Se calcularon las odds ratios (OR) ajustadas por todas las variables con los correspondientes intervalos de confianza al 95\% (IC 95\%). Se utilizó la técnica de regresión logística utilizando el paquete estadístico SPSS para Windows.

\section{RESULTADOS}

Los resultados presentados corresponden a los 755 sujetos entrevistados en 1996 $(64,5 \%$ del total de la población entrevistada inicialmente), de los cuales fallecieron $55(7 \%)$ durante el seguimiento efectuado en el período de 1996-1999. En la tabla 1 se describen las características sociodemográficas y la necesidad de ayuda para el cuidado personal y las actividades de la vida diaria de la muestra de 1996, según el sexo. En líneas generales, entre las mujeres había una proporción superior de personas de más de 75 años $(24,8 \%$ frente a un $17,7 \%)$, viudas $(47,2 \%$ frente $14,2 \%)$ y que vivían solas (33,3\% frente al 10,8\% entre los hombres). Por otro lado, una proporción superior declaraba un peor estado de salud percibida (56,3\% vs 40,2\% en las categorías de regular, mala o muy mala) y declaraba necesitar ayuda en el cuidado personal o en las actividades de la vida diaria.

En la tabla 2 se resumen las características más destacadas del apoyo social. Entre las mujeres una proporción superior declaraba no tener ningún apoyo vecinal ni apoyo emocional, siendo las diferencias estadísticamente significativas en el primer caso. La proporción de mujeres que declaraba no tener ningún amigo, ningún hijo, ningún sobrino o ningún hermano era superior a la declarada por los hombres, aunque las diferencias únicamente eran significativas en el caso de hijos y sobrinos. Con respecto a las organizaciones e instituciones sociales y religiosas, los hombres declaraban pertenecer a algún club o asociación y asistir habitualmente a los centros de jubilados y a las plazas del barrio con mayor frecuencia, mientras que las mujeres asistían con mayor frecuencia a la iglesia.

En la tabla 3 se presenta el análisis de la mortalidad para los hombres. En el análisis bivariado únicamente la edad y la necesidad de ayuda para las actividades cotidianas y el cuidado personal se asociaron significativamente con la mortalidad, mientras que convi- 
Tabla 1

Características sociodemográficas, salud percibida y necesidad de ayuda de la muestra de 1996, según el sexo

\begin{tabular}{|c|c|c|c|c|c|c|c|}
\hline & \multicolumn{2}{|c|}{ Hombres } & \multicolumn{2}{|c|}{ Mujeres } & \multicolumn{2}{|c|}{ Total } & \multirow{2}{*}{$p$. } \\
\hline & $N$ & $\%$ & $N$ & $\%$ & $n$ & $\%$ & \\
\hline \multicolumn{8}{|l|}{ Edad } \\
\hline $60-64$ & 93 & 29,4 & 122 & 27,8 & 215 & 28,5 & \\
\hline $65-69$ & 96 & 30,4 & 117 & 26,7 & 213 & 28,2 & \\
\hline $70-74$ & 71 & 22,5 & 91 & 20,7 & 162 & 21,5 & \\
\hline 75 y más & 56 & 17,7 & 109 & 24,8 & 165 & 21,9 & 0.03 \\
\hline \multicolumn{8}{|l|}{ Estado civil } \\
\hline Soltero/a & 10 & 3,2 & 43 & 9,8 & 53 & 7,0 & \\
\hline Casado/a & 256 & 81,0 & 183 & 41,7 & 439 & 58,1 & \\
\hline Viudo/a & 45 & 14,2 & 207 & 47,2 & 252 & 33,4 & \\
\hline Separado/a* & 5 & 1,6 & 4 & 0,9 & 9 & 1,2 & \\
\hline \multicolumn{8}{|l|}{ Situación de convivencia } \\
\hline Vive solo/a & 34 & 10,8 & 148 & 33,3 & 182 & 24,2 & \\
\hline Vive con su pareja & 184 & 58,6 & 133 & 30,4 & 317 & 42,2 & \\
\hline Vive con hijos & 63 & 20,1 & 46 & 10,5 & 109 & 14,5 & \\
\hline Vive con otras personas & 33 & 10,6 & 111 & 25,4 & 144 & 19,1 & $<0,001$ \\
\hline \multicolumn{8}{|l|}{ Salud percibida } \\
\hline Muy buena & 31 & 12,0 & 32 & 8,1 & 63 & 9,6 & \\
\hline Buena & 124 & 47,9 & 141 & 35,5 & 265 & 40,4 & \\
\hline Regular & 87 & 33,6 & 182 & 45,8 & 269 & 41,0 & \\
\hline Mala & 14 & 5,4 & 38 & 9,6 & 52 & 7,9 & \\
\hline Muy mala & 3 & 1,2 & 4 & 1,0 & 7 & 1,1 & 0,001 \\
\hline \multicolumn{8}{|c|}{$\begin{array}{l}\text { Necesidad de ayuda en el cuidado per- } \\
\text { sonal }\end{array}$} \\
\hline Sí & 21 & 6,6 & 52 & 11,8 & 73 & 9,7 & 0,017 \\
\hline \multicolumn{8}{|c|}{$\begin{array}{l}\text { Necesidad de ayuda en actividades co- } \\
\text { tidianas }\end{array}$} \\
\hline Sí & 40 & 12,7 & 129 & 29,4 & 169 & 22,4 & $<0,001$ \\
\hline Total & 316 & 100 & 439 & 100 & 755 & 100 & \\
\hline
\end{tabular}

vir con familiares $u$ otras personas pero no con la pareja mostró una asociación elevada (OR 3,7), aunque no significativa estadísticamente. Para las mujeres (tabla 4), tanto la edad como la situación de convivencia, la ala salud percibida (OR 5,7; IC 1,3-25,4) la falta de apoyo vecinal (OR 5,3; IC =1,8-15,3), la no asistencia habitual a la iglesia (OR 5,2; $\mathrm{IC}=2,1-12,6)$, el menor número de amigos y familiares (OR 5,4; IC=2,4-12,3), además de la necesidad percibida de ayuda en el cuidado personal y en las actividades cotidianas, se asociaron de forma significativa a la mor- talidad en el análisis bivariado, aunque en el análisis multivariado únicamente la edad y la falta de apoyo vecinal mantuvieron la significación estadística.

\section{DISCUSIÓN}

Los resultados del estudio permiten caracterizar el patrón de apoyo social entre las personas mayores en un entorno urbano, y muestran un efecto protector sobre la mortalidad general en las mujeres. En líneas gene- 
Tabla 2

Características del apoyo y red social de la muestra de 1996, según sexo

\begin{tabular}{|c|c|c|c|c|c|c|c|}
\hline \multirow{3}{*}{\begin{tabular}{|l} 
\\
Apoyo vecinal
\end{tabular}} & \multicolumn{2}{|c|}{ Hombre } & \multicolumn{2}{|c|}{ Mujer } & \multicolumn{2}{|c|}{ Total } & \multirow{2}{*}{$p$} \\
\hline & \multirow[t]{2}{*}{$N$} & \multirow[t]{2}{*}{$\%$} & \multirow[t]{2}{*}{$N$} & \multirow[t]{2}{*}{$\%$} & \multirow[t]{2}{*}{$n$} & \multirow[t]{2}{*}{$\%$} & \\
\hline & & & & & & & \\
\hline Nadie & 55 & 21,8 & 91 & 23,5 & 146 & 22,8 & \\
\hline 1 persona/familia & 28 & 11,1 & 84 & 21,7 & 112 & 17,5 & \\
\hline 2 o más & 169 & 67,1 & 212 & 54,8 & 381 & 58,6 & 0,002 \\
\hline \multicolumn{8}{|l|}{ Apoyo emocional } \\
\hline Nadie & 57 & 22,3 & 68 & 17,3 & 125 & 19,2 & \\
\hline 1 o más personas & 199 & 77,7 & 325 & 82,7 & 524 & 80,8 & 0,168 \\
\hline \multicolumn{8}{|l|}{ N. ${ }^{\circ}$ de amigos } \\
\hline 0 & 58 & 21,6 & 99 & 26,6 & 157 & 24,5 & \\
\hline 1 o más & 210 & 78,4 & 273 & 73,3 & 483 & 75,5 & 0,149 \\
\hline \multicolumn{8}{|l|}{ N. ${ }^{0}$ de hijos } \\
\hline 0 & 21 & 6,9 & 48 & 11,8 & 69 & 9,7 & \\
\hline 1 o más & 283 & 93,1 & 358 & 88,2 & 641 & 90,3 & 0,029 \\
\hline \multicolumn{8}{|l|}{ N. ${ }^{0}$ hermanos } \\
\hline 0 & 50 & 17,9 & 77 & 19,9 & 127 & 19,1 & \\
\hline 1 o más & 230 & 82,1 & 309 & 80,1 & 539 & 80,9 & 0,498 \\
\hline \multicolumn{8}{|l|}{ N. ${ }^{\circ}$ sobrinos } \\
\hline 0 & 27 & 9,9 & 60 & 15,7 & 87 & 13,3 & \\
\hline 1 o más & 246 & 90,1 & 321 & 84,3 & 567 & 86,7 & 0,030 \\
\hline \multicolumn{8}{|l|}{ N. ${ }^{\circ}$ de nietos } \\
\hline 0 & 48 & 16,9 & 64 & 16,4 & 112 & 16,6 & \\
\hline 1 o más & 236 & 83,1 & 326 & 83,6 & 562 & 83,4 & 0,866 \\
\hline \multicolumn{8}{|c|}{$\begin{array}{l}\text { Asistencia a la iglesia } \\
\text { (veces/semana) }\end{array}$} \\
\hline Ninguna vez & 242 & 76,6 & 266 & 60,6 & 508 & 67,3 & \\
\hline 1 o más & 74 & 23,4 & 173 & 39,4 & 247 & 32,7 & $<0,001$ \\
\hline \multicolumn{8}{|c|}{$\begin{array}{l}\text { Asistencia a centro de jubilados } \\
\text { (veces/semana) }\end{array}$} \\
\hline Ninguna vez & 278 & 88,0 & 408 & 92,9 & 686 & 90,9 & \\
\hline 1 o más & 38 & 12,0 & 31 & 7,1 & 69 & 8,1 & 0,015 \\
\hline \multicolumn{8}{|c|}{$\begin{array}{l}\text { Asistencia a plaza/lugar céntrico barrio } \\
\text { (veces/semana) }\end{array}$} \\
\hline Ninguna vez & 134 & 42,4 & 161 & 36,7 & 295 & 39,1 & \\
\hline 1 o más & 182 & 57,6 & 278 & 63,3 & 460 & 60,9 & 0,048 \\
\hline
\end{tabular}

rales, una de cada cinco personas de la muestra declara no tener ningún apoyo vecinal y una proporción similar declara no tener ningún apoyo emocional. Sólo un 25\% declara no tener amigos, y la mayoría manifiesta tener parientes de primer grado. Con respecto a las redes no familiares, menos del
$10 \%$ acuden habitualmente al centro de jubilados, y casi una tercera parte asisten regularmente al culto religioso, mientras que un $60,9 \%$ acuden habitualmente a la plaza, mercado o algún lugar céntrico del barrio. Con respecto a la asociación de las diversas variables con la mortalidad a los 3 años, vi- 
Tabla 3

Análisis bivariado y multivariado de la mortalidad para los varones

\begin{tabular}{|c|c|c|c|c|c|c|c|}
\hline & \multirow{2}{*}{$\begin{array}{c}\text { Total } \\
N\end{array}$} & \multicolumn{2}{|c|}{ Fallecidos } & \multirow{2}{*}{$O R$} & \multirow{2}{*}{ IC 95\% } & \multirow{2}{*}{ ORa } & \multirow{2}{*}{ IC $95 \%$} \\
\hline & & $n$ & $\%$ & & & & \\
\hline \multicolumn{8}{|l|}{ Edad } \\
\hline $60-74$ & 193 & 12 & 5,9 & 1 & & 1 & \\
\hline Más de 74 & 94 & 17 & 15,3 & 2,9 & $1,3-6,3$ & 1,1 & $0,5-2,3$ \\
\hline \multicolumn{8}{|l|}{ Situación de convivencia } \\
\hline Pareja & 231 & 20 & 8,0 & 1 & & 1 & \\
\hline Solos autónomos & 29 & 2 & 6,5 & 0,8 & $0,2-36$ & 0,5 & $0,1-2,8$ \\
\hline Solos dependientes & 22 & 7 & 24,1 & 3,7 & $0,7-9,5$ & 2,7 & $0,8-9,7$ \\
\hline \multicolumn{8}{|l|}{ Salud percibida } \\
\hline Muy buena/ buena & 147 & 8 & 5,2 & 1 & & 1 & \\
\hline Muy mala, mala o regular & 92 & 12 & 11,5 & 2,4 & $0,9-6,1$ & 1,9 & $0,7-5,4$ \\
\hline \multicolumn{8}{|l|}{ Apoyo vecinal } \\
\hline Alguien & 184 & 13 & 6,6 & 1 & & & \\
\hline Nadie & 50 & 5 & 9,1 & 1,4 & $0,5-4,2$ & & \\
\hline \multicolumn{8}{|l|}{ Apoyo emocional } \\
\hline Alguien & 186 & 13 & 6,5 & 1 & & 1 & \\
\hline Nadie & 50 & 7 & 12,3 & 2,0 & $0,8-5,3$ & 2,2 & $0,8-6,2$ \\
\hline \multicolumn{8}{|c|}{$\begin{array}{l}\text { Asistencia a iglesia, club de jubilados } \\
\text { o lugar céntrico }^{\text {(a) }}\end{array}$} \\
\hline Alguna vez & 167 & 15 & 8,2 & 1 & & & \\
\hline Ninguna vez & 120 & 14 & 10,4 & 1,3 & $0,6-2,8$ & & \\
\hline \multicolumn{8}{|l|}{ Tamaño red familiar ${ }^{(b)}$} \\
\hline Más de 5 familiares o amigos & 252 & 22 & 8,0 & 1 & & 1 & \\
\hline 5 o menos & 35 & 7 & 16,7 & 2,3 & $0,9-5,7$ & 3,1 & $0,9-10,0$ \\
\hline \multicolumn{8}{|c|}{$\begin{array}{l}\text { Necesidad ayuda actividades vida dia- } \\
\text { ria }\end{array}$} \\
\hline No & 256 & 19 & 6,9 & 1 & & 1 & \\
\hline Sí & 31 & 9 & 22,5 & 3,9 & $1,6-9,4$ & 1,9 & $0,5-7,4$ \\
\hline \multicolumn{8}{|c|}{ Necesidad ayuda cuidado personal } \\
\hline No & 271 & 24 & 8,1 & 1 & & & \\
\hline Sí & 16 & 5 & 23,8 & 3,5 & $1,2-10,5$ & & \\
\hline
\end{tabular}

(a) Al menos semanal; incluye amigos, hermanos, hijos, nietos y sobrinos (ver Métodos); OR: odds ratio bivariada; ORa: odds ratio ajustado.

vir sin pareja y con otros familiares, no tener apoyo emocional, y tener pocos amigos y familiares se asoció con la mortalidad en el análisis bivariado, pero no en el análisis multivariado para los varones. Para las mujeres, la mortalidad fue superior entre las que no vivían con su pareja, las que tenían menos apoyo vecinal, las que tenían menos amigos o familiares, o no acudían regularmente a la iglesia, lugar céntrico del barrio o centro de jubilados, aunque en el análisis multivariado únicamente se mantuvo la asociación con la falta de apoyo vecinal.

Entre las limitaciones del estudio cabe señalar, en primer lugar, los potenciales sesgos de selección, ya que un $20,8 \%$ de la muestra de 1992, elegida de forma aleatoria entre toda la población empadronada no institucionalizada, no pudo ser localizada en 
Tabla 4

Análisis bivariado y multivariado de la mortalidad para las mujeres

\begin{tabular}{|c|c|c|c|c|c|c|c|}
\hline & \multirow{2}{*}{$\begin{array}{c}\text { Total } \\
N\end{array}$} & \multicolumn{2}{|c|}{ Muerto } & \multirow{2}{*}{$O R$} & \multirow{2}{*}{ IC $95 \%$} & \multirow{2}{*}{ ORa } & \multirow{2}{*}{ IC $95 \%$} \\
\hline & & $N$ & $\%$ & & & & \\
\hline \multicolumn{8}{|l|}{ Edad } \\
\hline $60-74$ & 249 & 7 & 2,7 & 1 & & 1 & \\
\hline Más de 74 & 164 & 19 & 10,4 & 4,1 & $1,7-10,0$ & 1,1 & $1,0-1,2$ \\
\hline \multicolumn{8}{|l|}{ Convivencia } \\
\hline Pareja & 177 & 3 & 1,7 & 1 & & 1 & \\
\hline Solos autónomos & 136 & 9 & 6,2 & 3,9 & $2,4-30,7$ & 1,7 & $0,4-7,9$ \\
\hline Solos dependientes & 96 & 14 & 12,7 & 8,6 & $1,0-14,7$ & 2,6 & $0,6-12,2$ \\
\hline \multicolumn{8}{|l|}{ Salud percibida } \\
\hline Muy buena/buena & 171 & 2 & 1,2 & 1 & & 1 & \\
\hline Muy mala, mala o regular & 210 & 14 & 6,3 & 5,7 & $1,3-25,4$ & 4,0 & $0,8-20,9$ \\
\hline \multicolumn{8}{|l|}{ Apoyo vecinal } \\
\hline Alguien & 290 & 6 & 2,0 & 1 & & 1 & \\
\hline Nadie & 82 & 9 & 9,9 & 5,3 & $1,8-15,3$ & 3,6 & $1,1-11,1$ \\
\hline \multicolumn{8}{|l|}{ Apoyo emocional } \\
\hline Alguien & 312 & 13 & 4,0 & 1 & & & \\
\hline Nadie & 65 & 3 & 4,4 & 1,1 & $0,3-4,0$ & & \\
\hline \multicolumn{8}{|c|}{$\begin{array}{l}\text { Asistencia a iglesia, club de jubilado } \\
\text { o lugar céntrico }^{(a)}\end{array}$} \\
\hline Alguna vez & 271 & 7 & 2,5 & 1 & & 1 & \\
\hline Ninguna vez & 142 & 19 & 11,8 & 5,2 & $2,1-12,6$ & 2,6 & $0,6-7,3$ \\
\hline \multicolumn{8}{|l|}{ Tamaño red familiar ${ }^{(\mathbf{b})}$} \\
\hline Más de 5 familiares o amigos & 349 & 13 & 3,6 & 1 & & 1 & \\
\hline 5 o menos & 64 & 13 & 16,9 & 5,4 & $2,4-12,3$ & 2,2 & $0,6-7,3$ \\
\hline \multicolumn{8}{|c|}{$\begin{array}{l}\text { Necesidad ayuda actividades vida dia- } \\
\text { ria }\end{array}$} \\
\hline No & 301 & 9 & 2,9 & 1 & & & \\
\hline Sí & 112 & 17 & 13,2 & 5,1 & $2,2-11,7$ & & \\
\hline \multicolumn{8}{|c|}{ Necesidad ayuda cuidado personal } \\
\hline No & 370 & 17 & 4,4 & 1 & & 1 & \\
\hline Sí & 43 & 9 & 17,3 & 4,5 & $1,9-10,8$ & 2,7 & $0,8-9,9$ \\
\hline
\end{tabular}

(a) Al menos semanal; incluye amigos, hermanos, hijos, nietos y sobrinos (ver Métodos); OR: odds ratio bivariada; ORa: odds ratio ajustado.

1996. Sin embargo estas pérdidas no mostraron diferencias estadísticamente significativas con respecto a los que respondieron $^{28}$. Por otro lado, no se pueden descartar por completo sesgos de información, ya que las variables de apoyo social no han sido validados previamente en nuestro medio. Finalmente, hay que tener en cuenta el escaso número de fallecimientos observados ( 55 casos) en el período de seguimiento, que li- mita la potencia estadística del análisis. A esta limitación puede haber contribuido la pérdida para el seguimiento de las defunciones ocurridas fuera de Catalunya.

En general, estos resultados son bastante concordantes con otros estudios realizados en nuestro país, aunque la proporción de personas mayores que viven solas es muy superior a la observada en Leganés 
$(11,2 \%)^{26}$ o en Córdoba $(14,1 \%)^{22}$, en tanto que en Barcelona representó casi un $25 \%$, probablemente en relación con el mayor índice de sobreenvejecimiento de Barcelona. Por el contrario, el 50\% de las personas mayores de Leganés declararon no tener amigos, mientras que en Barcelona esta proporción fue del 24,5\%. En relación con la ayuda recibida, el porcentaje de personas que necesitaron ayuda para realizar actividades relacionadas con el cuidado personal fue del $18 \%$ en Leganés, del 16,6\% en Córdoba y del $9,7 \%$ en Barcelona; y para actividades relacionadas con el mantenimiento del ambiente: del $86 \%$, del $77 \%$ y del $22,4 \%$, respectivamente. Estas diferencias podrían reflejar la tendencia observada en los países desarrollados hacia un aumento progresivo de población anciana que vive sola ${ }^{29}$, tendencia que sería más acusada en zonas urbanas.

De los componentes del apoyo social analizados en estas investigaciones, el estado de convivencia no se asoció con la mortalidad en algunos estudios ${ }^{8,10}$, pero sí en $\operatorname{otros}^{1-3 ; 7-8 ; 10 ; 13}$. En el nuestro, el estado de convivencia mostró una asociación significativa con la mortalidad en ambos sexos, pero sólo en el análisis bivariante. Es decir, las personas que no estaban casadas pero que vivían con alguien (hijos, sobrinos, nietos, etc.) presentaron un mayor riesgo de fallecimiento. El apoyo emocional, que representa fundamentalmente la figura del confidente, esto es, la persona con quien poder hablar de problemas personales, se ha estudiado más extensamente en relación con la sintomatología depresiva $a^{30-32}$ y el estado de salud percibido ${ }^{2 ; 23 ; 33-36}$ que con la mortalidad. No obstante, en los estudios relacionados específicamente con la mortalidad, algunos autores encontraron asociación entre ambas variables $7,17,37$. En nuestro estudio, el mayor apoyo emocional no se asoció a la mortalidad en el análisis general ni tampoco al estratificar por sexos. Es probable que las diferencias de estos resultados obedezcan al tipo de variables incluidas en los estudios y a la variación de las escalas de medición empleadas: cualitativas o cuantitativas, así como a factores de tipo sociocultural, o factores de tipo psicosocial ${ }^{38-41}$. El apoyo instrumental, o la ayuda recibida por las personas mayores para la realización de actividades relativas al cuidado personal y a la vida diaria, se asoció en nuestro estudio a la mortalidad en hombres y en mujeres, tanto en el análisis bivariante como en el multivariante. Estos resultados coinciden plenamente con algunos estudios ${ }^{8 ; 17}$ y parcialmente con los de Avlund ${ }^{13}$, que encontró asociación en el caso de las mujeres, pero no en el de los hombres.

El apoyo vecinal, como componente de la red social, fue la única variable de nuestro estudio que se asoció en el análisis de regresión logística con la mortalidad, pero sólo en las mujeres. Algunos autores no distinguen entre vecinos y amigos, por lo que es difícil valorar la influencia de esta variable en los resultados obtenidos en sus investigaciones. En el estudio de Jylhä et $\mathrm{al}^{6}$ sí se especifican con claridad las relaciones con vecinos, aunque dicha variable no resultó ser predictora de la mortalidad. El tamaño de la red social (medido por el número de familiares y amigos, los vínculos familiares, los contactos o visitas con parientes y amigos) se asoció con la mortalidad en múltiples estudios ${ }^{1 ; 3-4 ; 8 ; 11 ; 16-17}$, aunque en otros, las asociaciones entre redes sociales y mortalidad, después de ajustar por las variables estudiadas, fueron débiles ${ }^{6 ; 12}$. La pertenencia a asociaciones, la asistencia a la iglesia, hogar del pensionista, plaza o lugar céntrico del barrio fueron componentes de la red social que se asociaron a la mortalidad en la mayoría de los estudios realizados ${ }^{1-2 ; 5-7 ; 10-11 ; 17 ; 37}$. En el nuestro, sólo se asociaron en el caso de las mujeres en el análisis bivariante.

En nuestro medio, parece ser que el apoyo social es más predictivo de la mortalidad en las mujeres, y que la red vecinal tiene mayor influencia que el número de amigos y familiares. Estos resultados deben interpretarse con cautela por las limitaciones señaladas, pero son consistentes en líneas generales 
con los estudios previos realizados en otros países que muestran un efecto protector del apoyo social sobre la mortalidad general. Los mecanismos causales deberán ser dilucidados en posteriores estudios y, probablemente, deberán ser establecidos a partir de la articulación de las influencias de contexto y la formulación de hipótesis muy específi$\operatorname{cas}^{20}$; en el caso de la población de mayor edad, probablemente se trate de una combinación de factores de motivación, información sobre recursos y efectos directos del apoyo social, como factores protectores principales ${ }^{9}$. En todo caso, y con independencia de los mecanismos causales, los resultados refuerzan la necesidad de identificar aquellos colectivos de mayor riesgo de aislamiento y la adopción de medidas e intervenciones preventivas, como fomentar los puntos y lugares de encuentro de todo tipo dirigidos a las personas ancianas y otros colectivos; aunque no es sostenible a medio plazo crear artificialmente redes sociales de apoyo, es posible y deseable fomentar a nivel social los lazos y vínculos de apoyo social, y dar apoyo a las personas y grupos en situación de riesgo ${ }^{42}$. Los beneficios en salud justificarán sin duda los esfuerzos.

\section{BIBLIOGRAFÍA}

1. Berkman LF, Syme SL. Social networks, host resistance, and mortality: a nine-year follow-up study of Alameda county residents. Am J Epidemiol 1979; 109: 186-204.

2. House JS, Robbins C, Metzner HL. The association of social relationships and activities with mortality: prospective evidence from the Tecumseh community health study. Am J Epidemiol $1982 ; 116 ; 1213-40$.

3. Schoenbach VJ, Kaplan BH, Fredman L, Kleinbaum DG. Social ties and mortality in Evans county, Georgia. Am J Epidemiol 1986; 123: 577-91.

4. Orth-GomerK, Johnson JV. Social netword interaction and mortality. J Chron Dis 1987; 40: 949-57.

5. Kaplan GA, Salonen JT, Cohen RD, Brand RJ, Syme L, Puska P. Social connections and mortality from all cuses and from cardiovascular disea- se: prospective evidence from eastern Finland. Am J Epidemiol 1988; 128:370-80.

6. Jylhä M, Aro S. Social ties and survival among the elderly in Tampere, Finland. Int J Epidemiol 1989; 18: 158-164.

7. Hanson BS, Isacsson SO, Janzon L, Lindell SE. Social network and social support influence mortality in elderly men. The prospective population study of «Men born in 1914» Malmö, Sweden. Am J Epidemiol 1989; 130:100-11.

8. Welin L, Svärdsudd K, Ander-Peciva S, Tibblin G, Tibblin B, Larsson B. Prospective study of social influences on mortality: the study of men born in 1913 and 1923. Lancet 1985; 1:915-8.

9. House JS. Landis KR, Umberson D. Social relationship and health. Science 1988; 241: 540-5 10.

10. Blazer DG. Social support and mortality in an elderly community population. Am J Epidemiol 1982; 115: 684-694.

11. Steinbach U. Social networks, institutionalisation, and mortality among elderley people in the United States. J Gerontol 1992; 47: 5183-5190.

12. Olsen J, Gunner- Svensson F, Waldstrom B. Social networks and longevity. A 14 year follow-up study among elderly in Denmark. Soc Sci 1991; 33: 1189-1195.

13. Avlund K, Damsgaard MT, Halstein BE. Social relations and mortality. An eleven year follow-up study of 70-year-old men and women in Denmark. Soc Sci Med 1998 Sep; 47 (5):635-43.

14. Baumann A, Filipiak B, Stieber J, Lowel H. Family status and social integration as predictors of mortality: a 5-year follow-up study of 55- to 74-year-old men and women in the Augsburg area. Gerontol Geriatr 1998 Jun; 31(3): 184-192.

15. Shye D, Mullooly JP, Freeborn DK, Pope CR. Gender differences in the relationship between social network support and mortality: a longitudinal study of an elderly cohort. Soc Sci Med 1995 Oct; 41(7): 935-47.

16. Yasuda N, Zimmerman SI, Hawkes W, Fredman L, Hebel JR, Mazinger J. Relation of social network characteristics to 5-year mortality among young-old versus old-old white women in an urban community. Am J Epidemiol 1997 Mar 15; 145(6): 516-23.

17. Pennin BW, van Tilburg T, Kriegsman DM, Deeg DJ, Boeke AJ, van Eijk JT. Effects of social support and personal coping resources on mortality in older age: the longitudinal Aging Study Amster- 
dam. Am J Epidemiol 1997 Sep 15; 146(6): 510-19.

18. Israel BA. Social networks and health status: linking theory, research, and practice. Patient counseling and health education $1982 ; 4: 65-79$.

19. Broadhead WE, Kaplan BH, James SA, Warner EH, Schoenbach VJ, Grimson R, Heyden S, Tibblin G, Gehlbach SH. The epidemiologic evidence for a relationship between social support and health. Am J Epidemiol 1983; 5: 521-37.

20. Berkman L.F., Glass T. (2000). Social integration, social networks, social support and health En: Berkman L.F., Kawachi I. (Ediciones). Socia Epidemiology (páginas 137-173). New York: Oxford University Press; 2000.

21. Béland F, Zunzunegui M. ${ }^{\mathrm{a}} \mathrm{V}$. Presentación de estudio envejecer en Leganés. Revista de gerontología. Número monográfico. 1995a Nov; 5.(4): 207-214.

22. Espejo J, Martínez de la Iglesia J, Rubio V, Dueñas R, Fernández MJ, Yun A. Recursos sociales en mayores de 60 años. Su relación con factores sociodemográficos y de salud (proyecto ANCO). Aten Primaria 1998; 21: 88-96.

23. Ferrando J, Nebot M, Borrell C, Egea L. Apoyo social y estado de salud percibido población no institucionalizada de más de 60 años. Gac Sanit 1996; 10: 174-182.

24. Borrell C, Arias A, Baranda L., Lozares C. Manual de l'enquesta de salut de Barcelona. Barcelona: Ajuntament de Barcelona; 1992.

25. Cerdà N, Nebot M, Borrell C. La xarxa social i la salut en la gent gran. Barcelona Societat 1996; 6 : $62-69$

26. Béland F, Zunzunegui MV. Envejecer en Leganés. Revista de Gerontología. Número monográfico. 1995b Nov; 5.(4): 209.

27. Silva LC. Excursión a la regresión logística en ciencias de la salud. Madrid: Díez de Santos; 1995.

28. Lafuente JM. Relación entre apoyo social y mortalidad en población anciana no institucionalizada. Estudio de seguimiento a los tres años. Bellvitge: Institut de Salut Pública; 2000.

29. Alvarez Dardet C, Peiró S. Informe SESPAS 2000: La salud pública ante los desafíos de un nuevo siglo. 4. a edición. Granada: Escuela Andaluza de Salud Pública; 2000.
30. Goldberg EL, Natta PV, Comstock GW. Depresive symtoms, social networks and social support of elderly women. Am J Epidemiol 1985;121: $448-56$

31. Grant I, Patterson TL, Yager J. Social supports in relation to physical health and symptoms of depression in the elderly. Am J Psychiatry 1988. $145: 1254-8$

32. Brown GW, Andrews B, Harris T, et al. Social Support, self esteem and depression. Psychol Med 1986;16: 813-31.

33. Weinberger M, Hiner SL, Tierney WM. Assessing social support in elderly adults. Soc Sci Med 1987;25:1049-1055,

34. Hanson BS, Östergren P-O. Social networks and health: theories and empirical evidence from Malmö, Sweden. Malmö: Lund University, Department of Community Health Sciences; 1994.

35. Dalgard OS, Bjork S, Tambs K. Social support, negative life events and mental health - a longitudinal study. Br J Psychiatry 1995; 166: 29-34

36. Kaplan GA, Wilson TW, Cohen RD, et al. Social functioning and overall mortality: prospective evidence from the Kuopio Ischemic Heart Disease Risk Factor Study. Epidemiology 1994; 5:495-500.

37. Seeman TE, Kaplan GA, Knudsen L, Cohen R, Guralnik J. Social networks ties and mortality among the elderly in the Alameda county study. Am J Epidemiol. 1987; 126: 714-23.

38. Dalgard OS, Haheim, LL. Psychosocial risk factors and mortality: a prospective study with special focus on social support, social participation, and locus of control in Norway. J Epidemiol Community Health 1998;52:476-481.

39. Liang J, Bennett JM, Krause NM, et al. Stress, social Relations, and Old Age Mortality in Taiwan. J Clin Epidemiol 1999; 52(10): 983-95.

40. Rosengren A, Orth-Gomer K, Wedel H, Whilhelmsen L. Stressful life events, social support, and mortality in men born in 1933. BMJ 1993 ;307: 1102-1105.

41. Johson JV, Stewart W, Hall EM, Fredlund P, Theorell T. Longterm psychosocial work environment and cardiovascular mortality among Swedish men. Am J Public Health 1996; 86 :324-31.

42. Stansfeld SA. Social support and social cohesión En. Marmot M, Wilkinson RG. Social determinants of health. Oxford: Oxford University Press; 1999.

Rev Esp Salud Pública 2002, Vol. 76, N. ${ }^{\circ} 6$ 